\title{
Organizational LCA: the new member of the LCA family - introducing the UNEP/SETAC Life Cycle Initiative guidance document
}

\author{
Julia Martínez-Blanco $^{1}$ Atsushi Inaba ${ }^{2} \cdot$ Ana $^{\text {Quiros }}{ }^{3}$. \\ Sonia Valdivia ${ }^{4} \cdot$ Llorenç Milà-i-Canals $^{5}$. \\ Matthias Finkbeiner ${ }^{1}$
}

Received: 29 April 2015 / Accepted: 26 May 2015 / Published online: 10 June 2015

(C) Springer-Verlag Berlin Heidelberg 2015

One of the flagship projects included in the phase III of the UNEP and SETAC's Life Cycle Initiative partnership is the so-called "LCA of organizations." It was kicked off in mid2013, and during the last 2 years, its nearly 70 participants have been drafting and validating the "Guidance on organizational life cycle assessment" (UNEP 2015). This editorial is the official announcement of the publication of this new UNEP/SETAC Life Cycle Initiative product and shortly presents it to the LCA community. Organizational LCA is a relevant and promising new member of the LCA family. Just like social LCA was promoted and supported by the publication of the Guidelines for Social LCA (UNEP/SETAC 2009), we expect that the guidance document now launched will serve making organizational LCA widely applied.

\section{The rationale behind organizational LCA}

Organizational LCA analyzes the whole organization (i.e., organizational approach), including not only the facilities of the organization but also upstream and downstream activities

Julia Martínez-Blanco

julia.martinezblanco@tu-berlin.de; julia.martinez@uab.cat

1 SEE, Department of Environmental Technology, Technische Universität Berlin, 10623 Berlin, Germany

2 Department of Environmental and Energy Chemistry, Kogakuin University, 163-8677 Tokyo, Japan

3 ECOGLOBAL, P.O.Box 134-1000, San José, Costa Rica

4 World Resources Forum, CH-9014 St. Gallen, Switzerland

5 UNEP-SETAC Life Cycle Initiative, 75009 Paris, France (i.e., life cycle approach) and considers a set of relevant environmental aspects (i.e., multi-impact approach). In this way, it provides organizations with environmental understanding at the level at which most of the decisions are made - the level of the organization - thus supporting them effectively to improve their environmental performance. Apart from the UNEP/SETAC Life Cycle Initiative flagship project, two main other initiatives exist on the development of this new approach, the ISO/TS 14072 (2014) and the OEF Guide (2013).

Over the past 10 years, organizational environmental analysis approaches have begun to emerge. They mostly consider the assessment of the full value chain as voluntary and concentrate on a single environmental aspect. However, the application and experience acquired by some of these worldwide known initiatives, like WRI and WBCSD (2004, 2011), have helped to promote the future use of LCA of organizations.

The LCA methodology is by definition a life cycle and multi-impact approach but was originally developed for products. The first efforts in the development of the LCA of organizations emerged in the 1990s (Klöpffer 1995; Taylor and Postlethwaite 1996; Finkbeiner et al. 1998; Clift and Wright 2000) and proposed hybrid approaches by combining input-output analysis and LCA. Recently, this new feature of LCA is becoming ever more relevant as a complement to product-based analyses. Product and organizational LCA are complementary in more than one way as they provide insights of an organization at different levels. Nevertheless, an LCA of an organization may be more complex, due to the multiple product life cycles involved and the fact that a large part of the environmental impacts can occur up and downstream. 


\section{What is organizational life cycle assessment?}

Organizational LCA $\left(\mathrm{OLCA}^{1}\right)$ is a compilation and evaluation of the inputs, outputs, and potential environmental impacts of the activities associated with the organization adopting a life cycle perspective (ISO 2014). Most of the principles and requirements of ISO 14040 and ISO 14044 for product LCA apply also for OLCA with some minor terminology amendments (Finkbeiner and König 2013). The major discrepancies between the two methodologies appear during scope definition and inventory assessment. OLCA requires the definition of two new elements, roughly equivalent to functional unit and reference flow, the so-called reporting organization and reporting flow. Another difference with product LCA is that OLCA is not recommended for comparative assertions intended to be communicated to the public. For further detail on this issue, see Martínez-Blanco et al. (2015a).

OLCA application may reveal environmental hotspots where the organization should focus energies and intervention, throughout the value chain and among all the products and operations involved in the provision of the portfolio. Understanding risk and impact reduction opportunities gives a solid ground to strategic decisions at different levels, for instance, when making decisions on technologies, investments, and new product lines. It may also serve as framework for tracking environmental performance over time and for informing corporate sustainability reporting.

\section{What is the scope of the new UNEP/SETAC Life Cycle Initiative publication?}

The new guidance document aims to be a publication accompanying the ISO 14072. The latter sets the basic requirements of the methodology, while the guidance document intends to provide further insight on the potential of the methodology and detailed recommendations that facilitate an easier and more widespread application of OLCA. At this point in time, proper guideline is available only for an environmental organizational LCA, even though the authors foresee the use of a similar approach for the social dimension of sustainability. The latter seems meaningful as social impacts mostly depend on the organizations' behavior and data is usually collected at the level of the organization.

${ }^{1}$ The publication "Guidance on organizational life cycle assessment" (UNEP 2015), which is presented in this editorial, uses the acronym "O-LCA" in analogy to other UNEP/ SETAC Life Cycle Initiative publications. However, according to the International Journal of Life Cycle Assessment editorial policy, the acronym used by ISO/TS 14072, without hyphen, is used here.
The publication has six main chapters that are close to 100 pages. Key guiding questions are included at the beginning of each chapter to facilitate in locating the relevant information. It is a comprehensive document with more than one target audience. Recommended reading itineraries are included and signaled along the document for decision makers, practitioners, methodology developers, and consumers or other stakeholders. Another feature of the publication are the "Boxes," which are dedicated to additional explanations, clarifications, or recommendations, and the "Reports" that support understanding of the main text by portraying real early case studies. Links with the relevant foundations for the definition of OLCA (WRI and WBCSD 2004, 2011; ISO 2006, 2014; European Commission 2013) are made throughout the guidance document.

Although complete and rigorous applications of OLCA are not yet a common practice, we identified first practical experiences of the use of approaches that encompass OLCA. Eleven experiences of the so-called "First Movers" are included in the guidance document to illustrate some methodological facets and the benefits that the methodology could bring to organizations. Most of these organizations developed their own adapted methodology. Eight sectors (hotel and catering, food, chemical, car manufacturing, energy, retail, consulting, and cosmetic and personal care) and four regions (South and North America, Europe, Asia, and Oceania) are represented in the case studies.

We devoted special efforts to make the new methodology operative for different types of organizations and in different situations. Organizations of all sizes, as well as every sector, have a key responsibility in the efforts to reduce environmental impacts. For example, large corporations play a promising role due to their relevant share of resources use and emissions generation in the global flows and their capacity to intervene, while medium and small organizations have an important contribution to the national economies and thus to overall environmental impacts, if addressed as a collective. The publication provides specific guidance for small- and medium-sized organizations by mainly simplifying the requirements and recommendations presented in the methodological framework.

Furthermore, previous knowledge is considered and used to ease the implementation of the methodology through the so-called "experience-based implementation pathways." These pathways provide particular methodological support according to the experience with other environmental tools (for example, product LCA or EMS) that the organization may have.

The guidance document is the first milestone result of a transparent development process, which included a group of 20 co-drafters and a stakeholder group of about 50 members from different sectors and backgrounds and from 31 different countries. Furthermore, many organizations and LCA experts, including the 11 "First Movers" presented at the guidance 
document, were contacted during the collection of examples (Martínez-Blanco et al. 2015b).

We would like to encourage the LCA community and also organization's managers and policy makers to apply and spread the methodology. The publication is publicly available at the website of the UNEP/SETAC Life Cycle Initiative. ${ }^{2} \mathrm{We}$ expect that it can move forward the integration of the environment in organizations' strategy and operation. The project has not finished with the launch of the guidance document. In order to test the publication, several case studies, from different regions and sectors and with different levels of experience on the use of environmental tools and data available, will be conducted after the release of the publication. We encourage you to download the guidance document, to apply it, and to explore the potential of organizational LCA. We hope for your support in promoting and empowering this new member of the LCA family.

Acknowledgments We would like to thank all the participants for their constructive inputs and feedback during the revision of the document and the 11 "First Movers" for their valuable contribution to the guidance document. We would like to particularly acknowledge the list of codrafters of the guidance document (in addition to the co-authors of this editorial): Jonas Bengtsson, Arthur Braunschweig, Bárbara María Cívit, Elisa Cobas Flores, Laura Draucker, Asela Kulatunga, Lise Laurin, Sven Lundie, Alessandro Manzardo, Manuele Margni, Toolseeram Ramjeawon, Sabrina Rodrigues Sousa, Jens Warsen, and Pia Alexandra Wiche Latorre. Finally, we thank the support of the ILCB and the secretariat of the UNEP/SETAC Life Cycle Initiative.

\section{References}

Clift R, Wright L (2000) Relationships between environmental impacts and added value along the supply chain. Technol Forecast Soc Chang 65:281-295

European Commission (2013) Organisation Environmental Footprint Guide. European Commission - Joint Research Centre - Institute for Environment and Sustainability, http://eur-lex.europa.eu/ JOHtml.do?uri=OJ:L:2013:124:SOM:EN:HTML
Finkbeiner M, König P (2013) Carbon footprint and life cycle assessment of organizations. J Environ Account Manag 1: $55-63$

Finkbeiner M, Wiedemann M, Saur K (1998) A comprehensive approach towards product and organisation related environmental management tools. Int J Life Cycle Assess 3:169-178

ISO (2006) ISO 14044: environmental management - life cycle assessment-requirements and guidelines. International Organization for Standardization, Geneva

ISO (2014) ISO/TS 14072: environmental management - life cycle assessment-requirements and guidelines for Organizational Life Cycle Assessment. International Organization for Standardization, Geneva

Klöpffer W (1995) Betriebsökobilanzen. Seminar Ökologische Produktion und EU-Öko-Audit. Haus der Technik, Essen

Martínez-Blanco J, Inaba A, Finkbeiner M (2015a) Scoping organizational LCA — challenges and solutions. Int J Life Cycle Assess 20(6): 829-841

Martínez-Blanco J, Inaba A, Finkbeiner M (2015b) Halfway point in the flagship project "LCA of organizations" by UNEP/SETAC Life Cycle Initiative. J Life Cycle Assess Jpn 11:1-7

Taylor AP, Postlethwaite D (1996) Overall business impact assessment (OBIA). Proc. 4th SETAC Case Study Symp. Brussels, Belgium Brussels, p 181-187

UNEP (2015) Guidance on Organizational Life Cycle Assessment. LifeCycle Initiative, United Nations Environment Programme and Society for Environmental Toxicology and Chemistry, Paris, France, http://www.lifecycleinitiative.org/wp-content/uploads/ 2015/04/o-lca_24.4.15-web.pdf

UNEP/SETAC (2009) Guidelines for social life cycle assessment of products. Life-Cycle Initiative, United Nations Environment Programme and Society for Environmental Toxicology and Chemistry, Paris, France, http://www.lifecycleinitiative.org/wp-content/uploads/ 2012/12/2009 - Guidelines for sLCA - EN.pdf

WRI and WBCSD (2004) GHG Protocol Corporate Accounting and Reporting Standard. World Resources Institute and World Business Council for Sustainable Development, http:// www.ghgprotocol.org/files/ghgp/public/ghg-protocol-revised. pdf

WRI and WBCSD (2011) Corporate value chain (Scope 3) accounting and reporting standard - supplement to the GHG protocol corporate accounting and reporting standard. World Resources Institute and World Business Council for Sustainable Development, http://www. ghgprotocol.org/files/ghgp/public/Corporate-Value-ChainAccounting-Reporing-Standard_041613.pdf

\footnotetext{
${ }^{2} \mathrm{http} / /$ www.lifecycleinitiative.org/wp-content/uploads/2015/ 04/o-lca_24.4.15-web.pdf.
} 